\title{
255 INVESTIGATING VISTA'S ROLE INTRINSIC TO T CELLS IN THE TUMOR MICROENVIRONMENT
}

Cassandra Gilmour*, Li Wang, Juan Dong, Sarah Stone, Keman Zhang, Hieu Ta. Cleveland Clinic Foundation, Cleveland, OH, USA

Background Cancer immunotherapies, specifically checkpoint blockade therapies, have demonstrated clinical importance for long term patient survival. One of the major limitations to checkpoint blockade therapies, is the low response rate: $\sim 30 \%$ with anti-CTLA4 and anti-PD1 treatment. This may be due to heterogeneity of the patients' immune system and the tumor microenvironment including $\mathrm{T}$ cell inhibitions. There is a clear need to study this phenomenon and develop additional therapies for long term survival to include a broad range of patients. V-domain Immunoglobulin Suppressor of T-cell Activation (VISTA) is a suppressive protein expressed on many cell types in the tumor microenvironment including cytotoxic $\mathrm{T}$ cells. VISTA's role on $\mathrm{T}$ cells has been described as maintaining quiescence and peripheral tolerance in a graft vs host disease model, but is not fully understood in context of the tumor microenvironment.

Methods We use a series of invivo experiments, including $\mathrm{T}$ cell specific VISTA knock outs, to understand the role of VISTA on T cells in the tumor microenvironment.

Results Here we show a series of in vivo experiments that suggest VISTA has a potent intrinsic role on T cells and therefore anti-tumor immunity. Using a T cell specific VISTA knock out, our results suggest that the absence of VISTA on T cells in combination with anti-CTLA4 and vaccine is a very powerful tumor suppressor compared to vaccine and anti-CTLA4 treatment alone. These results also indicate that the absence of VISTA alters the phenotype of cytotoxic $\mathrm{T}$ cells in several ways including the production of inflammatory cytokines.

Conclusions Our preliminary data provides foundation to study VISTA's role intrinsic to $\mathrm{T}$ cells in the tumor microenvironment and how disrupting VISTA's influence intrinsic to $\mathrm{T}$ cells may be advantageous for anti-tumor immunity and long term patient survival.

Ethics Approval All in vivo studies were reviewed and approved by Institutional Animal Care and Use Committee (Approval number 2019-2142).

http://dx.doi.org/10.1136/jitc-2021-SITC2021.255 